\title{
Topical and Conventional Systemic Treatments in Atopic Dermatitis: Have They Gone Out of Fashion?
}

\author{
Giulia Calabrese $^{1}$, Gaetano Licata ${ }^{1}$, Alessio Gambardella ${ }^{1}$, Alina De Rosa ${ }^{1}$, Roberto Alfano ${ }^{2}$, \\ Giuseppe Argenziano ${ }^{1}$
}

1 Dermatology Unit, Department of Mental and Physical Health and Preventive Medicine, University of Campania Luigi Vanvitelli, Naples, Italy

2 Department of Anesthesiology Surgery and Emergency, University of Campania Luigi Vanvitelli, Naples, Italy.

Keywords: atopic dermatitis, treatments, therapy, topicals, systemic therapy

Citation: Calabrese G, Licata G, Gambardella A, De Rosa A, Alfano R, Argenziano G. Dermatol Pract Concept. 2022;12(1):e2022155. DOI: https://doi.org/10.5826/dpc.1201a155

Accepted: January 7, 2022; Published: January 2022

Copyright: $@ 2022$ Calabrese et al. This is an open-access article distributed under the terms of the Creative Commons

Attribution-NonCommercial License (BY-NC-4.0), https://creativecommons.org/licenses/by-nc/4.0/, which permits unrestricted noncommercial use, distribution, and reproduction in any medium, provided the original authors and source are credited.

Funding: None.

Competing interests: None.

Authorship: All authors have contributed significantly to this publication.

Corresponding author: Giulia Calabrese, MD, Dermatology Unit, Department of Mental and Physical Health and Preventive Medicine, University of Campania Luigi Vanvitelli, E-mail: allergologiavanvitel-li@gmail.com

\footnotetext{
This article is part of a series of reviews dedicated to Atopic Dermatitis, guest edited by Prof Anna Balato.

Guest Editor

Prof. Anna Balato, MD, PhD

Associate Professor of Dermatology, Dermatology Unit, University of Campania, Naples, Italy
}

\begin{abstract}
Atopic dermatitis is a Th2 disease, due to relapse of IL-4 and IL-13 by Th2 cells. Despite the approval by FDA of dupilumab, the first monoclonal antibody for the severe forms, traditional drugs remain a milestone for the treatment of this dermatosis. Dermatologists need a good knowledge of all therapies for an integrated and personalized management of patients.
\end{abstract}

\section{Introduction}

Atopic dermatitis $(\mathrm{AD})$ is a chronic, inflammatory skin disease, characterized by a multifactorial pathogenesis. AD is considered a prototypical Th2 disease, due to relapse of IL-4 and
IL-13 by Th2 cells. IL-4 and IL-13 are involved in the survival of Th2 cells, differentiation and activation of myeloid and dendritic cell, B-cell maturation, stimulation of IgE class switching, eosinophil chemotaxis and down-regulation of genes involved in skin barrier function (fillagrin, locrin, and involucrin). 
Conventional treatments represent a cornerstone of $\mathrm{AD}$ therapy and are the first-line therapy for the mild-moderate forms. With biological drugs rapidly emerging, it is important for clinicians to combine the new treatments with the traditional ones to improve the response of the patients [1-4,17].

\section{Conventional therapy for $\mathrm{AD}$}

Conventional treatments for $\mathrm{AD}$ can be differentiated in topical and systemic. Topical agents are moisturizers, corticosteroids, calcineurin inhibitors and antimicrobials/antiseptics, used in monotherapy or in combination. In the field of topical therapies, wet wrap therapy must also be mentioned [5-9].

Systemic treatments, used for severe and recalcitrant forms, consist in phototherapy, systemic corticosteroids, cyclosporine, methotrexate, mycophenylate mofetil and azathioprine [10].

\section{Moisturizers}

Moisturizers are used to combat xerosis (one of the cardinal clinical features of $\mathrm{AD}$ and the result of a dysfunctional epidermal barrier) and transepidermal water loss. They reduce disease severity, the need for pharmacologic intervention and should be applied soon after bathing, almost once a day [5-7].

Moisturizers can be classified in traditional (glycol and glyceryl stearate, soy sterols, petrolatum, dimethicone, mineral oil, glycerol, lactic acid, urea) and prescription emollient devices (PEDs). Traditional agents contain varying amounts of emollient, occlusive and humectant ingredients. Emollients (eg glycol and glyceryl stearate, soy sterols) lubricate and soften the skin, occlusive agents (eg petrolatum, dimethicone, mineral oil) form a layer to reduce evaporation of water, while humectants (.g glycerol, lactic acid, urea) attract and hold water. Several clinical trials have shown that they lessen symptoms and signs of $\mathrm{AD}$, including pruritus, erythema, fissuring, and lichenification [5-7].

The term PED has been introduced to identify a new class of topical agents designed to target the specific defects in skin barrier function observed in AD. They include preparations having distinct ratios of lipids that mimic endogenous compositions and containing palmitoylethanolamide, glycyrrhetinic acid, or other hydrolipids. PEDs may contain an antioxidant agent, such as furfuryl palmitate or furfuryl derivatives. PEDs lessen xerosis and inflammation but they have been tested in a small number of controlled studies [11].

Moisturizers are the main primary treatment for mild forms and should be part of the regimen for moderate and severe ones because they reduce inflammation, the prescription of topical corticosteroids and prevent the flares in the maintenance treatment. There are few studies that have compared moisturizers, but results are overlapping. Therefore, the choice of moisturizing agent is highly dependent on individual preference [5].

In $\mathrm{AD}$, the alteration of skin barrier is associated with reduced ceramide levels, with a consequential increase in trans-epidermal water loss (TEWL) and reduction in skin hydration.

It is not yet known whether dupilumab restores skin barrier function. The latter can be investigated using TEWL (that is the water evaporization rate in $\mathrm{g} / \mathrm{h} / \mathrm{m}^{2}$ ) and stratum corneum hydration $(\mathrm{SCH})$ (the water content in the stratum corneum measured by Corneometer ${ }^{\circledR}$ ).

According to a recent study, following treatment with dupilumab, TEWL decreases quickly in the skin interested by $\mathrm{AD}$, while $\mathrm{SCH}$ does not improve. Because of the small sample size in this study, further papers are needed but it seems that dupilumab have no effects for dry skin conditions and patients have to continue the use of moisturizers [12].

\section{Topical corticosteroids}

Topical corticosteroids (TCS) inhibit the antigen processing and suppress the release of pro-inflammatory cytokines, acting on a variety of immune cells, including $\mathrm{T}$ lymphocytes, monocytes, macrophages, and dendritic cells. Their efficacy has been demonstrated, with more than 110 different randomized controlled trials [5]. Twice daily application of corticosteroids is generally recommended for the treatment of $\mathrm{AD}$; however, evidence suggests that once daily application may be sufficient. As maintenance therapy to reduce the number of relapses, a "proactive" approach can be prescript. It consists of applying TCS intermittently once or twice a week in the areas most prone to relapse, even when no inflammatory lesions are visible. The CHRONOS study examined the use of dupilumab combined with topical corticosteroids versus placebo in 52 weeks of treatment. Thanks to the combination with topical corticosteroids, $10 \%$ of patients more than in the SOLO studies (that have tested just dupilumab versus placebo) achieved a $75 \%$ reduction in EASI score [13].

\section{Wet Wrap Therapy}

The wet wrap therapy (WWT) is a method to quickly reduce $\mathrm{AD}$ severity, used for significant flares and recalcitrant disease. It consists in the application of topical agent (TCS or moisturizers or combination of both) covered by a wetted first layer of tubular bandages, gauze, or a cotton suit, followed by a second dry layer.

WWT increases the penetration of topical therapy and reduces the water loss. The wrap can be worn from several hours to 24 hours at a time, depending on patient tolerance 
and the application can be repeated for several days up to 2 weeks. The major limitation of this technique is represented by the lack of practicality and the low tolerance for patients, especially if children $[8,9]$.

\section{Topical Calcineurin Inhibitors}

Topical calcineurin inhibitors (TCIs) inhibit calcineurin-dependent T-cell activation, blocking the production of pro-inflammatory cytokines. Two types of TCIs are available: tacrolimus ointment $(0.03 \%$ and $0.1 \%$ strengths $)$ and pimecrolimus cream ( $1 \%$ strength). TCIs are recommended and effective for acute and chronic treatment, twice a day, as well as maintenance, in both adults and children with AD. They can be used as steroid-sparing agents in long term therapy because they do not cause cutaneous atrophy and can be used in sensitive areas, such as the face, skin folds and genitals. Tacrolimus $0.03 \%$ ointment and pimecrolimus cream are indicated for use in individuals aged 2 years and older, while tacrolimus $0.1 \%$ strength is only approved in those over age 15 years. Proactive, intermittent use of TCIs as maintenance therapy (2-3 times a week) on areas that commonly flare is recommended to prevent relapses and is more effective than the use of emollients alone. This strategy has been tested for up to 1 year, without significant adverse events. The most common side effects are local reactions such as stinging and burning. TCIs may be combined with TCS [5].

For patients undergoing dupilumab injections, tacrolimus ointment and pimecrolimus cream may be helpful to treat facial eczema (that responds later to dupilumab) and peripalpebral dermatitis (as a prevention of dupilumab associated conjunctivitis). Conjunctivitis is the most common side effect of dupilumab [14]. The prescription of trehalose/ hyaluroate tear substitute and the treatment of peripalpebral and facial eczema with topical therapy reduce the incidence of dupilumab-associated conjunctivitis (incidence equal to $5 \%$ with preventive therapy versus an incidence of $8 \%$ and $8-23 \%$ without preventive therapy, according to trial studies and real life studies respectively) [15]. Moreover, new emerging problems are reported in literature: recalcitrant (pre-existing biological therapy) and dupilumab-associated (probably induced by biological therapy) face and neck dermatitis are described and can benefit by topical therapy (antifungal-corticosteroids and tacrolimus ointment). Various etiopathogenic mechanisms for these new regional dermatoses have been proposed but more studies are needed [16].

\section{Phototherapy}

Phototherapy is used as second-line treatment, after failure of first-line treatment (emollients, topical steroids, and TCIs), or as maintenance therapy in patients with chronic disease.
Multiple forms of light therapy are beneficial for disease and symptom control, including: natural sunlight, narrowband (NB) UVB, BB-UVB, UVA, topical and systemic PUVA. Home phototherapy under the direction of a physician may be considered for patients who are unable to receive phototherapy in a medical office setting.

The light modality chosen should be guided by factors such as availability, cost, patient skin type, skin cancer history, and patient use of photosensitizing medications (for example calcineurine inhibitor). The dosing and scheduling of light should be based on minimal erythema dose and Fitzpatrick skin type. Phototherapy is usually administered 3 times a week.

NB-UVB is generally the most commonly recommended light treatment, considering its low risk profile and the relative efficacy. Several common adverse effects include: actinic damage, local erythema and tenderness, pruritus and burning [10].

\section{Systemic Corticosteroids}

The management of $\mathrm{AD}$ with systemic corticosteroids should generally be avoided because of short- and long-term adverse effects. Their use should be exclusively reserved for acute, severe exacerbations and as a short-term bridge therapy to other systemic, steroid-sparing therapy [10].

\section{Cyclosporine}

Cyclosporine (CSA) is an immunosuppressant of T-cells and interleukin-2 production, used for adult and pediatric patients with severe or refractory form of AD. The dosage of CSA (available in oral capsules and solution) ranges from 3 to $6 \mathrm{mg} / \mathrm{kg} / \mathrm{d}$, standardly 150 to $300 \mathrm{mg} /$ day in adults, divided in two doses. The initial and maintenance dose of CSA should be based on multiple factors, including the patient's disease severity and other medical morbidities. Potential adverse effects include: hypertension, infection, nephrotoxicity, hypertension, tremor, hypertrichosis, headache, gingival hyperplasia, and increased risk of skin cancer and lymphoma. Thus, patients receiving CSA should be monitored for such potential consequences. The recommended time limit for consecutive use of CSA is currently 1 year. Before starting therapy, the following tests should be prescribed: blood count, renal and liver function, lipids, electrolytes, uric acid, hepatitis B and C markers, HIV and human chorionic gonadotropin (HCG) if indicated. During therapy, blood pressure should be measured twice a week and blood tests should be tested monthly [10]. CSA can be associated with dupilumab in the initial phase of therapy to induce faster the clinical remission or in patients recalcitrant to dupilumab in monotherapy [17]. 


\section{Methotrexate}

Methotrexate (MTX) is an antifolate metabolite and blocks the synthesis of DNA, RNA, and purines. It is also thought to negatively affect T-cell function. Its many off-label uses include treatment of refractory AD. MTX is available in solution (for intramuscular or subcutaneous injection) and oral tablet form. MTX is usually given as a single weekly dose. The dose range for MTX in patients with AD is extrapolated from its use in psoriasis and it is between 7.5 and $25 \mathrm{mg}$ weekly. Folate supplementation is recommended during treatment with MTX. Nausea and other gastrointestinal symptoms may preclude oral administration. Severe adverse effects, including bone-marrow suppression and pulmonary fibrosis, can occur. Literature suggests that bone-marrow suppression is often reversible upon MTX dose reduction or discontinuation. Risk for skin cancer and lymphoma has been reported, although some cases of lymphoma arising during low-dose treatment have regressed on drug discontinuation. Pulmonary fibrosis may occur with short- or long-term use of the medication, such that patients with pulmonary diseases (eg asthma, chronic cough) may not be candidates. Before starting therapy, the following tests should be prescribed: blood count, renal and liver function, lipids, electrolyte, uric acid, hepatitis B and C markers, HIV and HCG if indicated. During the therapy, blood tests should be tested monthly. There are no data about use of MTX in children affected by AD. Multiple studies regarding its use in pediatric patients with psoriasis show MTX as a safe, effective, and well-tolerated medication [10]. Like cCSA, MTX can be associated with dupilumab in recalcitrant patients, maintaining a good safety profile [17].

\section{Azathioprine}

Azathioprine (AZA) is a purine analog that inhibits DNA production, thus preferentially affecting cells with high proliferation rates, such as B-cells and T-cells during inflammatory disease states. It is used off-label to treat inflammatory cutaneous and systemic disorders, including refractory $\mathrm{AD}$ with moderate improvement of dermatitis (equal to a $37 \%$ reduction of disease, measured by the Six Area, Six Sign AD scoring system after 12 weeks) [18]. A delayed effect may be noted, with some patients needing a treatment for 12 weeks or longer to achieve full clinical benefit. The dose range is variable between 1 to $3 \mathrm{mg} / \mathrm{kg} / \mathrm{d}$. AZA is available in tablet form only and may be given once daily.

Graduated dosing to maximize benefit while limiting side effects is preferred, as a considerable number of patients develops intolerable nausea and vomiting at higher doses. The metabolism of AZA is dependent on an individual's thiopurine S-methyltransferase (TPMT) activity level, a principal enzyme in the thiopurine pathway. Genetic polymorphisms in TPMT activity are linked to a patient's susceptibility to AZA toxicity, such that the homozygous carrier state of low or absent enzyme capacity poses the greatest toxicity risk. Thus, baseline TPMT level testing is strongly recommended before AZA initiation, with avoidance of use in those with very low or absent enzyme activity. Concomitant phototherapy is not advised because of increased risk of DNA damage and possible photocarcinogenicity. Nausea, vomiting and other gastrointestinal symptoms (bloating, anorexia, cramping) are common adverse effects. Other side effects include headache, hypersensitivity reactions, elevated liver enzymes, and leukopenia. AZA can be used for pediatric population in case of recalcitrant dermatitis but there are not sufficient data to recommend an optimal dose, duration of therapy, or to predict the relapse rate upon discontinuation. However, the most common dosage given is $2.5 \mathrm{mg} / \mathrm{kg} / \mathrm{d}$, with a higher treatment range maximum of $4 \mathrm{mg} / \mathrm{kg} / \mathrm{d}$ relative to adult dosing (maximum $3 \mathrm{mg} / \mathrm{kg} / \mathrm{d})$ [10].

\section{Mycophenolate Mofetil}

Mycophenolate mofetil (MMF) is an immunosuppressant that blocks the purine biosynthesis pathway of cells via the inhibition of inosine monophosphate dehydrogenase, selectively affecting B- and T-cells. It is recognized as an off-label therapy in patients with refractory forms of AD. Insufficient data exist to make recommendations regarding the optimal MMF dosing or duration of therapy for patients with AD. Dosing ranges from 0.5 to $3 \mathrm{~g} / \mathrm{d}$. MMF is available in oral suspension, capsules, and tablets, and it is given twice daily. MMF is generally well tolerated, with nausea, vomiting, and abdominal cramping being the most common side effects. Rarely, hematologic (anemia, leukopenia, thrombocytopenia) and genitourinary (urgency, frequency, dysuria) toxicities have been reported. There is a theoretical risk of increased susceptibility to viral and bacterial infections while taking MMF, as it is clearly observed in patients with organ transplantation. Cutaneous malignancy and lymphoma are potential risks [10].

\section{Conclusions}

The introduction of dupilumab has changed the management of patients with severe forms of $\mathrm{AD}$ but traditional drugs remain a milestone for the treatment of mild-moderate $\mathrm{AD}$ and for combined therapies. Therefore, dermatologists need a good knowledge of both fields of interest for an integrated and personalized management of patients. 
Table 1. Key points

\begin{tabular}{|l|l|}
\hline Moisturizers & $\begin{array}{l}\text { Integral part of AD therapy, including in patients on biological treatment since it is able to reduce } \\
\text { inflammation and itching but not xerosis. }\end{array}$ \\
\hline $\begin{array}{l}\text { Topical } \\
\text { corticosteroids }\end{array}$ & $\begin{array}{l}\text { Recommended for mild-to-moderate forms; they can be also helpful to accelerate the response to } \\
\text { systemic drugs, including biologics, to increase the efficacy and to control the flares. }\end{array}$ \\
\hline $\begin{array}{l}\text { Topical } \\
\text { calcineurin } \\
\text { inhibitors }\end{array}$ & $\begin{array}{l}\text { Recommended as steroid-sparing agents in long term therapy and mostly for the sensitive areas (face, } \\
\text { skin folds and genitals). Useful to treat facial dermatitis (not responsive to dupilumab or dupilumab } \\
\text { associated) and to prevent dupilumab associated conjunctivitis, if peripalpebral eczema is present. }\end{array}$ \\
\hline Phototherapy & $\begin{array}{l}\text { Used as second-line treatment, after failure of first-line treatment (emollients, topical steroids, and } \\
\text { topical calcineurin inhibitors), or as maintenance therapy in patients with chronic disease. }\end{array}$ \\
\hline $\begin{array}{l}\text { Traditional } \\
\text { systemic drugs }\end{array}$ & $\begin{array}{l}\text { First line therapy for severe forms and a valid choice in combination with dupilumab and in case of } \\
\text { inadequate response to biological drug, maintaining a good safety profile. }\end{array}$ \\
\hline
\end{tabular}

\section{References}

1. Blauvelt A, de Bruin-Weller M, Gooderham M, et al. Longterm management of moderate-to-severe atopic dermatitis with dupilumab and concomitant topical corticosteroids (LIBERTY AD CHRONOS): a 1-year, randomised, double-blinded, placebo-controlled, phase 3 trial. Lancet. 2017;389 (10086): 2287-2303. DOI: 10.1016/S0140-6736(17)31191-1. PMID: 28478972.

2. Silverberg JI, Yosipovitch G, Simpson EL, et al. Dupilumab treatment results in early and sustained improvements in itch in adolescents and adults with moderate to severe atopic dermatitis: Analysis of the randomized phase 3 studies SOLO 1 and SOLO 2, AD ADOL, and CHRONOS. J Am Acad Dermatol. 2020;82(6):1328-1336. DOI: 10.1016/j.jaad.2020.02.060. PMID: 32135208.

3. Chiricozzi A, Maurelli M, Gori N, et al. Dupilumab improves clinical manifestations, symptoms, and quality of life in adult patients with chronic nodular prurigo. J Am Acad Dermatol. 2020;83(1):39-45. DOI: 10.1016/j.jaad.2020.03.049. PMID: 32229281.

4. Ribero S, Giura MT, Viola R, et al. Effectiveness and safety of dupilumab for the treatment of atopic dermatitis in adult cohort: a real-life Italian tertiary centre experience. J Eur Acad Dermatol Venereol. 2020;34(8):e380-e383. DOI: 10.1111/jdv.16219. PMID: 31960496.

5. Eichenfield LF, Tom WL, Berger TG, et al. Guidelines of care for the management of atopic dermatitis: section 2. Management and treatment of atopic dermatitis with topical therapies. J Am Acad Dermatol. 2014;71(1):116-32. DOI: 10.1016/j. jaad.2014.03.023. PMID: 24813302. PMCID: PMC4326095.

6. Rawlings AV, Canestrari DA, Dobkowski B. Moisturizer technology versus clinical performance. Dermatol Ther. 2004;17 Suppl 1:49-56. DOI: 10.1111/j.1396-0296.2004.04s1006.x. PMID: 14728699 .

7. Draelos ZD. An evaluation of prescription device moisturizers. J Cosmet Dermatol. 2009;8(1):40-43. DOI: 10.1111/j.14732165.2009.00422.x. PMID: 19250165.

8. Dabade TS, Davis DM, Wetter DA, et al. Wet dressing therapy in conjunction with topical corticosteroids is effective for rapid control of severe pediatric atopic dermatitis: experience with 218 patients over 30 years at Mayo Clinic. J Am Acad Dermatol. 2012;67(1):100-6. DOI: 10.1016/j.jaad.2011.06.025. PMID: 21978575 .
9. Devillers AC, Oranje AP. Efficacy and safety of 'wet-wrap' dressings as an intervention treatment in children with severe and/or refractory atopic dermatitis: a critical review of the literature. Br J Dermatol. 2006;154(4):579-585. DOI: 10.1111/j.13652133.2006.07157.x. PMID: 16536797.

10. Sidbury R, Davis DM, Cohen DE, et al. Guidelines of care for the management of atopic dermatitis: section 3. Management and treatment with phototherapy and systemic agents.J Am Acad Dermatol. 2014;71(2):327-349. DOI: 10.1016/j.jaad.2014.03.030. PMID: 24813298. PMCID: PMC4410179.

11. Nolan K, Marmur E. Moisturizers: reality and the skin benefits. Dermatol Ther. 2012;25(3):229-233. DOI: 10.1111/j.15298019.2012.01504.x. PMID: 22913439.

12. Furuhashi T, Oda T, Torii K, Nishida E, Morita A. Dupilumab probably reduces transepidermal water loss but does not increase stratum corneum hydration in atopic dermatitis. J Dermatol. 2021;48(2):e74-e75. DOI: 10.1111/1346-8138.15638. PMID: 33035368 ; PMCID: PMC7891664.

13. Blauvelt A, de Bruin-Weller M, Gooderham M, et al. Long-term management of moderate-to-severe atopic dermatitis with dupilumab and concomitant topical corticosteroids (LIBERTY AD CHRONOS): a 1-year, randomised, double-blinded, placebocontrolled, phase 3 trial. Lancet. 2017;389(10086):2287-2303. DOI: 10.1016/S0140-6736(17)31191-1. PMID: 28478972.

14. Treister AD, Kraff-Cooper C, Lio PA. Risk Factors for DupilumabAssociated Conjunctivitis in Patients With Atopic Dermatitis. JAMA Dermatol. 2018;154(10):1208-1211. DOI: 10.1001/jamadermatol.2018.2690. PMID: 30167653. PMCID: PMC6233741.

15. Calabrese G, Gambardella A, Licata G, Di Brizzi EV, Alfano R, Argenziano G.Dupilumab and conjunctivitis: a case series of twenty patients. J Eur Acad Dermatol Venereol. 2021;35(9):e612-e614. DOI: $10.1111 /$ jdv.17210. PMID: 33657247.

16. Jaros J,HendricksAJ,ShiVY,LioPA.A PracticalApproach to Recalcitrant Face and Neck Dermatitis in Atopic Dermatitis. Dermatitis. 2020;31(3):169-177. DOI: 10.1097/DER.0000000000000590. PMID: 32355092.

17. Gori N, Chiricozzi A, Malvaso D, et al. Successful Combination of Systemic Agents for the Treatment of Atopic Dermatitis Resistant to Dupilumab Therapy. Dermatology. 2021;237(4):535541. DOI: 10.1159/000512890. PMID: 33477153.

18. Meggitt SJ, Gray JC, Reynolds NJ. Azathioprine dosed by thiopurine methyltransferase activity for moderate-to-severe atopic eczema: a double-blind, randomised controlled trial. Lancet. 2006; 367:839846. DOI: 10.1016/S0140-6736(06)68340-2. PMID: 16530578. 